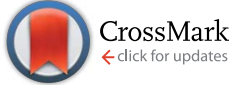

Cite this: RSC Adv., 2017, 7, 10051

Received 31st October 2016 Accepted 30th January 2017

DOI: $10.1039 / c 6 r a 26063 b$

rsc.li/rsc-advances

\section{Fabrication of a silver octahedral nanoparticle- containing polycaprolactone nanocomposite for antibacterial bone scaffolds $\uparrow$}

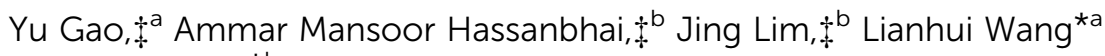 \\ and Chenjie $\mathrm{Xu}$ *bc
}

\begin{abstract}
This work investigated the antimicrobial activity of Ag octahedral nanoparticle containing polycaprolactone scaffolds (Ag-PCL) that is fabricated via cryomilling. The fabricated composite scaffolds exhibited localized antibacterial activity with no adverse effects on viability and osteogenic differentiation of human fetal mesenchymal stem cells (hfMSCs). Compared to plain PCL scaffolds, the Ag-PCL scaffolds significantly reduce bacteria survival to $32.2 \%$ over a 4 hour incubation.
\end{abstract}

\section{Introduction}

The frequency and relevance of age-related diseases, such as healing and regeneration of bone tissue, are rising due to increasing life expectancy. When bone defects exceed a critical size, impaired bone formation can occur and surgical intervention becomes mandatory. ${ }^{\mathbf{1 , 2}}$ The development of biomaterials and stem cell therapy spurred the success of bone tissue engineering with the goal of regenerating bone tissue on porous scaffolds. Bone tissue engineered (BTE) scaffolds provide not only mechanical support but also an osteoconductive environment that allow the regeneration of bone with improved mechanical properties. ${ }^{3-5}$

Infections during or post scaffold transplantation are still challenging which compromise the efficacy of bone healing. ${ }^{6,7}$ For example, bacteria can adhere to scaffold surfaces before and during transplantation, then form a protective biofilm that competes with the host for integration with the scaffold. ${ }^{8}$ After transplantation, infections can also spread to bone scaffold from other sources of inflammation through bloodstream. ${ }^{9}$ Upon development of infection, surgical debridement of infected tissue and antibiotic treatment is often used. The integration of controlled antibiotic release to BTE scaffold represent a more attractive manner as it can regenerate the

${ }^{a}$ Key Laboratory for Organic Electronics and Information Displays, Institute of Advanced Materials (IAM), Jiangsu National Synergistic Innovation Center for Advanced Materials (SICAM), Nanjing University of Posts \& Telecommunications, 9 Wenyuan Road, Nanjing 210023, China.E-mail: iamlhwang@njupt.edu.cn

${ }^{b}$ School of Chemical and Biomedical Engineering, Nanyang Technological University, 70 Nanyang Drive, Singapore 637457. E-mail: cjxu@ntu.edu.sg

${ }^{c}$ NTU-Northwestern Institute for Nanomedicine, Nanyang Technological University, 50 Nanyang Avenue, Singapore 639798

$\dagger$ Electronic supplementary information (ESI) available. See DOI: 10.1039/c6ra26063b

\$ These author contributed equally to this work. bone, and simultaneously control the infection at the localized location. Various antibiotic (e.g. tetracycline, ciprofloxacin and gentamycin) containing BTE scaffolds have been fabricated through dip-coating, ${ }^{\mathbf{1 0}}$ freeze drying ${ }^{\mathbf{1 1}}$ and compression moulding. ${ }^{12}$ Most of them exhibited an initial burst followed by a sustained antibiotic release, and proved to be effective for killing bacterial in vitro.

Silver nanoparticles, a new generation of antimicrobials, have been emerged up in the present scenario owing to the need of non-drug antimicrobial agents. ${ }^{13}$ This is because the miss-use and over use of antibiotics worldwide that cause increase of occurrence of antibiotic resistance. The nanosized Ag particles possess large surface area to volume ratio which provide better interaction with bacteria. The major contribution of antimicrobial effects of silver nanoparticles was attributed to the released $\mathrm{Ag}$ ions in most studies. ${ }^{\mathbf{1 3 , 1 4}}$ The $\mathrm{Ag}$ ions can bind to the cell membrane associated proteins leading to membrane damage. ${ }^{15}$ The disruption of DNA replication and inhibition of respiratory chain in bacteria proved to be other mechanisms of action of $\mathrm{Ag}$ ions. ${ }^{16,17}$ It was reported that $\mathrm{Ag}$ triangular nanoplates with more $\{111\}$ facets exhibit more efficient antibacterial activities than Ag spherical (mainly $\{100\}$ facets) or rod shaped ( $\{100\}$ and $\{111\}$ facets) nanoparticles. ${ }^{18}$ Similar results were also found in the research of $\mathrm{Cu}_{2} \mathrm{O}$ nanoparticles, in which octahedral crystals bounded by $\{111\}$ facets exhibited higher antibacterial activities than cubic ones (bounded by $\{100\}$ facets). ${ }^{19}$

In this work, Ag octahedral nanoparticles (mainly $\{111\}$ facets) were synthesised and their antibacterial activity was studied. Later, Ag octahedral nanoparticles were integrated into polycaprolactone (PCL) scaffold by cryomilling to achieve homogenous distribution. The fabricated composite scaffolds exhibited localized antibacterial activity with no adverse effects on viability and osteogenic differentiation of human fetal mesenchymal stem cells (hfMSCs). 


\section{Results and discussions}

Synthesis, characterization and antibacterial effect of $\mathbf{A g}$ octahedral nanoparticles

Ag octahedral nanoparticles were synthesized using the polyol reduction method, which started first with the synthesis of $\mathrm{Ag}$ nanocubes. ${ }^{\mathbf{2 0 2 1}}$ They were stabilized by polyvinylpyrrolidone (PVP) and stocked in ethanol before use. The synthesized Ag octahedron exhibited clear polyhedral structure with high productivity and homogeneous size distribution (Fig. 1A). By measuring the particle size in scanning electron microscopy (SEM) images, its diameter was determined as $328 \pm 18 \mathrm{~nm}$ (Fig. 1B). UV/Vis spectra was obtained after the nanoparticle solution was repeatedly washed with ethanol to remove excess polymer and filtered. The extinction spectra (extinction = scattering + absorption) of $\mathrm{Ag}$ octahedral nanoparticles in Fig. 1C exhibited highly complex plasmon signature due to their geometric anisotropy. The spectrum in the range 400 to $600 \mathrm{~nm}$ revealed the fine structure and was assigned to hexapolar and higher-order modes, while the resonance after $800 \mathrm{~nm}$ were mainly quadrupolar mode. These findings confirmed the structure of synthesized Ag octahedral nanoparticles and were in good agreement with reported works. ${ }^{20}$

The antimicrobial assay of $\mathrm{Ag}$ octahedral nanoparticles were performed on a Gram-negative bacteria, pseudomonas aeruginosa PAO1, which is known as a prototypical multidrug resistant (MDR) pathogen. Ag octahedral nanoparticles $\left(40 \mu \mathrm{g} \mathrm{mL}^{-1}\right)$



Fig. 1 Characterization of Ag octahedrons. (A) Representative scanning electron microscopy image of synthesized Ag octahedral nanoparticles. Scale bar, $1 \mu \mathrm{m}$. (B) Size distribution of Ag octahedral nanoparticles $(n>500)$ measured from SEM images. (C) Extinction spectra of Ag octahedral nanoparticles.

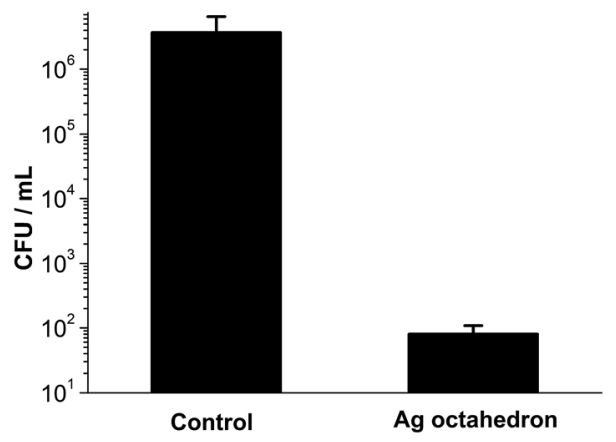

Fig. 2 Antimicrobial assay of Ag octahedron ( $40 \mu \mathrm{g} \mathrm{mL}^{-1}$ ) cultured with pseudomonas aeruginosa PAO1 for 4 hours.

were added to the bacteria with a starting inoculum of $2 \times 10^{4}$ CFU $\mathrm{mL}^{-1}$. After 4 hours incubation, bacteria were counted by spread plate method. As shown in Fig. 2, Ag octahedral nanoparticles at the concentration of $40 \mu \mathrm{g} \mathrm{mL}^{-1}$ exhibited effective antimicrobial activity with a decrease of bacteria survival (80 CFU $\mathrm{mL}^{-1}$ ) by five orders of magnitude in comparison to control group with no addition of silver nanoparticles $\left(3.7 \times 10^{6}\right.$ CFU mL $\left.{ }^{-1}\right)$.

After validating the antibacterial effect of $\mathrm{Ag}$ octahedral nanoparticles against pseudomonas aeruginosa, we set to incorporate these nanoparticles into polymeric scaffold. PCL has been widely used in biodegradable scaffolds and drug containing implants for tissue engineering and cancer therapy. ${ }^{22-25}$ As PCL is a hydrophobic polymer, dispersing hydrophilic Ag nanoparticles within the hydrophobic matrix is challenging. Fortunately, we have developed a method to fabricate inorganic nanoparticle (e.g. tricalcium phosphate or silica) containing PCL composites with homogeneous distribution of nanoparticles by cryomilling. ${ }^{22-24}$ This physical and solvent-free method was carried out at low temperature $\left(-196{ }^{\circ} \mathrm{C}\right)$ that also protect the active ingredients such as $\mathrm{Ag}$ nanoparticles against oxidation. Indeed, the fabricated Ag-PCL film exhibited even light brown color (the color of Ag octahedrons), whereas pure PCL (figure below, left) exhibited white color (ESI Fig. S1†).

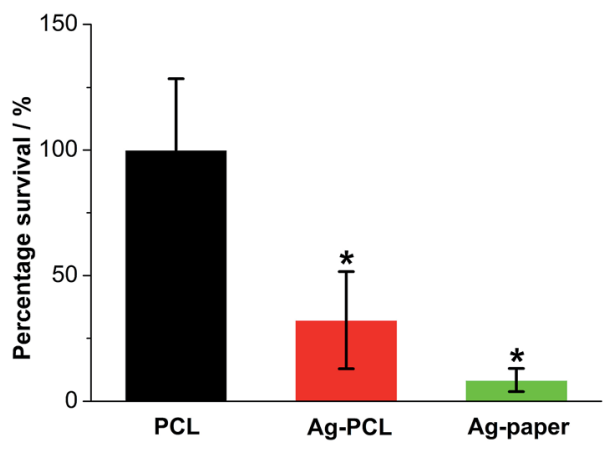

Fig. 3 Antimicrobial assay of PCL film scaffold, Ag octahedral nanoparticle containing PCL scaffold, and Ag octahedral nanoparticle containing filter paper against pseudomonas aeruginosa PAO1 after 4 hours culturing. Statistical significance was determined by calculating the $P$-value using the Student's $t$-test (two-tailed distribution and twosample unequal variance). ${ }^{*}$ denotes $p<0.05, N=3$. 


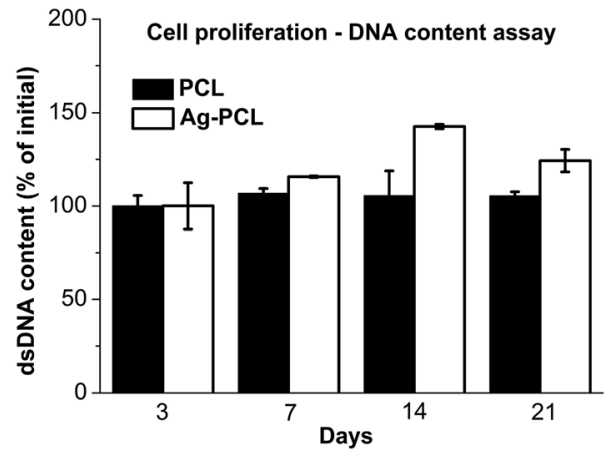

Fig. 4 hfMSCs proliferation on PCL or Ag-PCL scaffolds examined by DNA quantities through Picogreen assay.

The fabricated composite PCL film scaffold contained 1\% (mass ratio) of Ag octahedral nanoparticles that was approximately 40 $\mu \mathrm{g}$ per film (12 $\mathrm{mm}$ in diameter and $200 \mathrm{~nm}$ in thickness) calculated by measuring the mass of each Ag-PCL film. Under this mass ratio, composite films allow sufficient cargo loading (Ag octahedrons) with not compromised mechanical properties compared to pure PCL film. ${ }^{22-24}$

\section{Antimicrobial assay of Ag octahedral nanoparticle containing PCL scaffold}

Later, Ag octahedral nanoparticle containing PCL (Ag-PCL) film scaffolds and plain PCL film scaffolds were sterilized and placed in a 12 well plate. $1 \mathrm{~mL}$ of pseudomonas aeruginosa solution $(2 \times$ $10^{4} \mathrm{CFU} \mathrm{mL}{ }^{-1}$ ) was added to each well and cultured for 4 hours. The unbounded bacteria were removed and the wells were washed by PBS twice. Then the attached bacteria in each well were collected by vortex and counted as mentioned above. According to the antimicrobial assay (Fig. 3), Ag-PCL scaffold inhibited the bacteria survival to $32.2 \%$ in comparison to that of plain PCL scaffold. This inhibition of survival was not as effective as that induced by the same amount of free Ag octahedral nanoparticles in Fig. 2 (almost 5 orders of magnitude inhibition). As shown in Fig. 3, when the same amount of Ag octahedral nanoparticles were soaked into filter papers (with same area to Ag-PCL or PCL scaffolds), the prepared filter papers (Ag-paper) exhibited more efficient antibacterial effect
( $8.4 \%$ of bacteria survival), owing to its faster dissociation rate of $\mathrm{Ag}$ ions and easier mass transfer of interior $\mathrm{Ag}$ ions. Note: filter paper has a porous structure and Ag nanoparticles all absorbed on the surface of the fibres in the filter paper.

This difference is because only surface bounded Ag nanoparticles in the Ag-PCL scaffold were exposed to the bacteria and surrounding medium. Most of the Ag octahedral nanoparticles will be released once the scaffold are undergoing degradation. It renders the Ag-PCL scaffold the ability of prolonged release of $\mathrm{Ag}$ ions with the scaffold degradation post transplantation, which is an attractive strategy for chronic inflammation often happened in a long period after surgery. ${ }^{26}$

When placing the Ag-PCL film scaffold on the surface of plate cultured with pseudomonas aeruginosa, localized inhibition effect was observed. Bacteria grew by the side of the film scaffold, while no bacteria was found on the scaffold surface (ESI Fig. S2 $\dagger$ ). This localized antibacterial effect was related to the amount of released Ag octahedral nanoparticles from the scaffold (ESI Fig. S3†). Limited nanoparticle release from PCL composite was also found in silica nanoparticle containing PCL film previously. ${ }^{24}$ This nature might be attractive to reduce the adverse effects of released $\mathrm{Ag}$ nanoparticles to surrounding healthy tissues. ${ }^{27}$

\section{hfMSCs proliferation and osteogenic differentiation on Ag- PCL scaffold}

Finally, the effects of incorporated Ag octahedral nanoparticles to hfMSCs proliferation and osteogenic differentiation were evaluated. hfMSCs were seeded on PCL and Ag-PCL film scaffolds and cultured for 21 days. The results of DNA quantification using Picogreen assay suggested that the attached cells were able to maintain viability and slowly proliferate on both PCL and Ag-PCL scaffold for 21 days (Fig. 4). The slow proliferation rate of hfMSCs on PCL scaffold in static culture was in good agreement with reported work, ${ }^{28}$ whereas a slightly faster proliferation rate was presented on Ag-PCL scaffold than that on PCL scaffold. The increased surface roughness of scaffold due to the ingredients (ESI Fig. S4 $\dagger$ ) that could influence the cell adhesion and proliferation, might be partly responsible for the increased proliferation rate of hfMSCs on the Ag-PCL film. ${ }^{29}$ In short, the embedded $\mathrm{Ag}$ octahedral nanoparticles (1\%) in Ag-PCL film did not
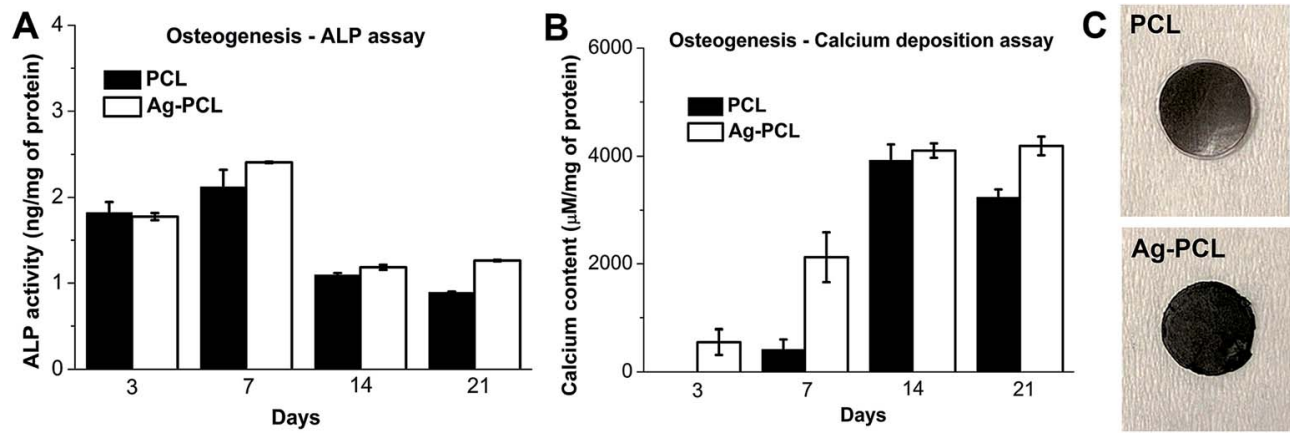

Fig. 5 hfMSCs osteogenic differentiation on both PCL and Ag-PCL film scaffolds. The efficiencies of osteogenic differentiation were evaluated by (A) ALP assay, (B) calcium deposition assay, and (C) von Kossa staining assay (day 21) respectively. 


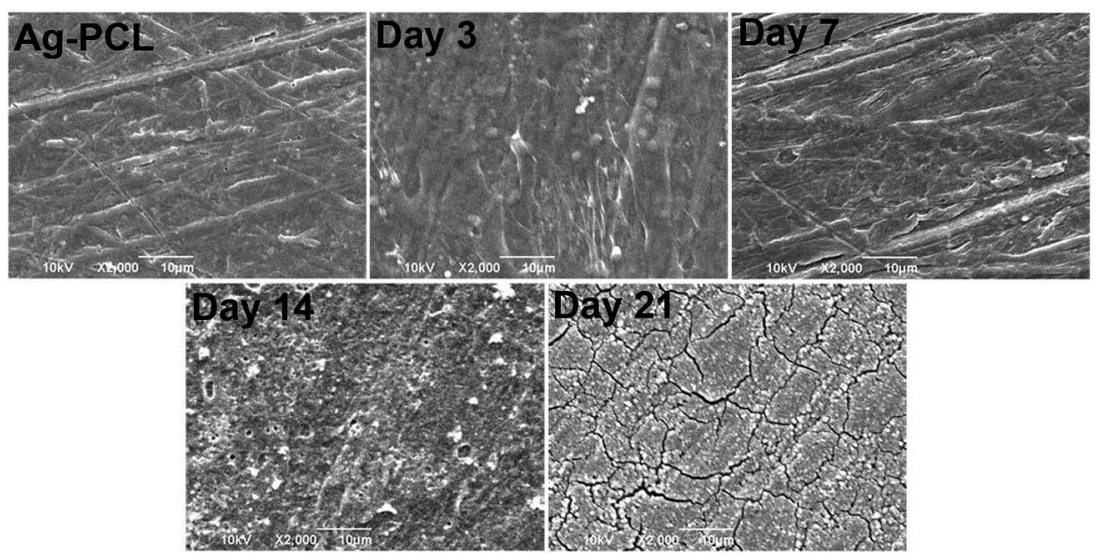

Fig. 6 SEM images of Ag-PCL film scaffold and hfMSCs loaded Ag-PCL film scaffolds that were induced by osteogenic differentiation for 3, 7, 14 , and 21 days respectively.

compromise the hfMSCs viability and proliferation seeded on the scaffold, comparing to that on PCL film scaffold.

Besides proliferation, the ability of osteogenic differentiation of hfMSCs was also evaluated by standard methods including alkaline phosphatase (ALP) expression, calcium deposition and von Kossa staining. The amount of ALP expression in differentiating hfMSCs on either PCL or Ag-PCL scaffold experienced an abrupt increase on day 7 , following a steadily decrease on day 14 and final stabilization at day 21 (Fig. 5A). The mineralization levels assessed by calcium deposition assay on both PCL and $\mathrm{Ag}$-PCL scaffolds underwent similar stepwise increases during day 3 to day 14, and achieved plateau on day 21 (Fig. 5B). The mineralization was further confirmed by von Kossa staining, presenting dark color on both PCL and Ag-PCL scaffolds (Fig. 5C). Similar results (ALP expression and calcium assay) for hfMSCs on PCL scaffolds were reported previously. ${ }^{30,31}$ Furthermore, the higher ALP expression and calcium deposition, and the darker von Kossa staining on Ag-PCL than PCL scaffolds in the present work were correlated with their higher DNA contents. Thus, the ingredients $(1 \%$ of $\mathrm{Ag}$ octahedral nanoparticles) in Ag-PCL film did not compromise the ability of hfMSCs osteogenic differentiation, suggesting its potential application on tissue engineered bone scaffolds.

SEM images revealed the morphological change of Ag-PCL scaffolds during osteogenesis for 21 days (Fig. 6). On day 3 and 7 , similar morphology of cell-loaded scaffold to scaffold without cells were presented. During day 14 to 21 , significant cellular matrix and calcium deposition presented on the scaffold, suggesting the different extent of mineralization with time (SEM images of PCL film incubated with medium only were presented in ESI Fig. S5†). These results confirmed the findings in ALP and calcium deposition assay, and indicated good biocompatibility of Ag-PCL scaffold on bone regeneration.

\section{Conclusions}

In summary, Ag octahedral nanoparticles were synthesized and exhibited effective antibacterial effects. Through cryomilling, these Ag octahedrons were successfully incorporated into PCL film scaffolds with homogeneous distribution. The fabricated Ag-PCL scaffold exhibited localized antibacterial effects with $32.2 \%$ inhibition of percentage survival to pseudomonas aeruginosa in comparison to plain PCL. Furthermore, no adverse effects on viability and osteogenic differentiation of hfMSCs on Ag-PCL film was observed. This Ag-PCL scaffold might hold great promise in bone tissue engineering with simultaneous infection control and minimal undesired side effects caused by excessive silver ions release.

\section{Experimental}

\section{Synthesis of $\mathrm{Ag}$ octahedral nanoparticles}

The preparation of Ag octahedron was carried out using the polyol reduction method, started first with the synthesis of Ag nanocubes. First, $10 \mathrm{~mL}$ of $\mathrm{CuCl}_{2}\left(8 \mathrm{mg} \mathrm{mL}^{-1}\right)$, PVP $(20 \mathrm{mg}$ $\left.\mathrm{mL}^{-1}\right)$, and $\mathrm{AgNO}_{3}\left(20 \mathrm{mg} \mathrm{mL}^{-1}\right)$ were dissolved in 1,5-pentanediol (PD) separately. $35 \mu \mathrm{L}$ of $\mathrm{CuCl}_{2}$ solution was added to the $\mathrm{AgNO}_{3}$ solution. $20 \mathrm{~mL}$ of PD was then heated to $190{ }^{\circ} \mathrm{C}$ for $10 \mathrm{~min} .250 \mu \mathrm{L}$ of PVP precursor was added to a round-bottom flask dropwise every $30 \mathrm{~s}$ while $500 \mu \mathrm{L}$ of $\mathrm{AgNO}_{3}$ precursor was injected into the flask every minute in one go. The reaction was allowed to proceed for approximately $20 \mathrm{~min}$. Following which, the injection was continued using $30 \mathrm{~mL}$ of PVP $\left(20 \mathrm{mg} \mathrm{mL}^{-1}\right)$ and $\mathrm{AgNO}_{3}\left(40 \mathrm{mg} \mathrm{mL}{ }^{-1}\right.$ with $120 \mu \mathrm{L}$ of $\mathrm{CuCl}_{2}$ ) were separately prepared in PD. The reaction was allowed to proceed until the precursors were used up. The Ag octahedron solution was redispersed in $20 \mathrm{~mL}$ of ethanol after removing the PD via multiple centrifugation rounds, and diluted, then vacuum filtered multiple times using PVDF filter membranes (Durapore $®$ ) with pore sizes ranging from $5000 \mathrm{~nm}, 650 \mathrm{~nm}$, $450 \mathrm{~nm}$ to $220 \mathrm{~nm}$ to remove impurities before finally dispersing and stocking in ethanol.

\section{Fabrication of Ag-PCL film scaffold}

Medical grade PCL pellets ( $>500 \mu \mathrm{m})$ (Osteopore International, Singapore) were weighed and placed into a stainless steel jar, before they were pulverized in a cryomilling process (Retsch ${ }^{\circledR}$, 
Germany) at low temperature cooled by liquid nitrogen. Then Ag octahedral nanoparticles and cryomilled PCL (mass ratio of $1: 100)$ were mixed and cryomilled again for 20 minutes to attain powdered Ag-PCL. The powders were then thermally pressed into a thin film between two stainless steel sheets at $80{ }^{\circ} \mathrm{C}$ (Carver Inc, USA). The film was then stored in a dry cabinet (Digi-Cabi AD-100, Singapore) before use.

\section{Bacteria culture}

Pseudomonas aeruginosa PAO1 was purchased from ATCC and used in this study. They were cultured in Mueller-Hinton agar and Mueller-Hinton broth and incubated at $37^{\circ} \mathrm{C}$.

\section{Antimicrobial assay}

PAO1 was grown overnight. The culture was diluted to achieve a starting inoculum of $2 \times 10^{4} \mathrm{CFU} \mathrm{mL}^{-1}$. In each well of a 12well plate, $1 \mathrm{~mL}$ of the culture solution was added to plain PCL, Ag-PCL and Ag-filter. For the free Ag, $40 \mu \mathrm{g}$ was added to the well. The plate was incubated for 4 hours. The samples are removed and washed twice in PBS to remove any unbounded bacteria. $1 \mathrm{~mL}$ of PBS is then added to the sample and vortexed for $2 \mathrm{~min}$ to remove all attached bacteria. Serial dilution is performed and counts obtained using the spread plate method CFU $\mathrm{mL}^{-1}=$ number of colonies $\times 10 \times$ dilution factor. For the free $\mathrm{Ag}$, counts are performed directly on the supernatant.

\section{Isolation and culture of hfMSCs}

hfMSC were isolated from fetal femurs as previously described..$^{28}$ Briefly, single-cell suspensions were prepared by flushing the marrow cells out of human fetal femurs using a $22 \mathrm{G}$ needle and passed through a $70 \mathrm{~mm}$ cell strainer and plated on a $75 \mathrm{~cm}^{2}$ cell culture flask (NUNC, USA) at $10^{6}$ cells per $\mathrm{mL}$ using Dulbecco's modified Eagle's medium (DMEM)Glutamax (GIBCO, USA) supplemented with 10\% fetal bovine serum (FBS, Hyclone), $50 \mathrm{U} \mathrm{mL}^{-1}$ penicillin and streptomycin (GIBCO, USA). MSCs of passage 4-6 were used for this study and they were not differentiated prior to seeding on scaffolds.

\section{Osteogenic differentiation}

Osteogenic induction medium was prepared by supplementing the culture media with $0.2 \mathrm{mM}$ ascorbic acid, $10 \mathrm{mM} \beta$-glycerophosphate and $10^{-8} \mathrm{M}$ dexamethasone (Sigma-Aldrich, USA) and sterile-filtered prior to use. These supplements were added to the culture medium and replaced every 3-4 days.

\section{Alkaline phosphatase activity assay}

The cell lysates were tested for ALP activity using SensoLyte ${ }^{\mathrm{TM}}$ pNPP Alkaline Phosphatase Assay Kit (AnaSpec, USA) according to manufacturer's instructions. The ALP activities were normalized to the total protein content determined using the Bradford assay.

\section{Bradford protein assay}

Coomassie reagent (Thermo-Fisher) was used. Here, the cell lysates of the samples were added to samples in a 96-well plate followed by addition of the reagent. After 15 minutes incubation at room temperature, the absorbance at $595 \mathrm{~nm}$ was read. The results were compared against the standard curve using bovine serum albumin (BSA).

\section{DNA content}

The DNA content indirectly provides an indication of cell numbers. Quantitative analysis of DNA using the cell lysates was performed using PicoGreen DNA Quantification Kit (Molecular Probes, USA) according to manufacturer's instructions.

\section{Calcium content}

The film samples were added to acetic acid (to dissolve the calcium) for overnight. The content was determined by a colorimetric assay using a calcium assay kit (BioAssay Systems, USA) according to manufacturer's instructions. The controls were films with-out cells.

\section{Von Kossa staining}

Film samples were fixed with $4 \%$ paraformaldehyde and thereafter washed thrice in distilled water. Samples were stained with $2 \%$ silver nitrate (Sigma-Aldrich, USA) for 10 minutes in the dark and then exposed to bright light for 15-30 minutes.

\section{Scanning electron microscope}

SEM images of Ag octahedral nanoparticles were captured by the scanning electron microscope (JSM-6390LA, Jeol, Japan). For film scaffold samples, they were fixed in $2 \%$ glutaraldehyde and subsequently washed in PBS. The samples were then subjected to a graded ethanol series of 50\%, 70\%, 95\% and 100\% for 15 minutes at each concentration. Hexamethyldisilazane (Sigma-Aldrich, USA) was added to the samples for 20 minutes and removed and allowed to air dry. Samples were then spluttercoated and viewed by SEM.

\section{Conflict of interest}

The authors declare no competing financial interests.

\section{Acknowledgements}

Y. Gao acknowledges the National Natural Science Foundation of China (81601608), Natural Science Foundation of Jiangsu Province of China (BK20160919), Natural Science Fund for Colleges and Universities in Jiangsu Province (16KJB310008), NUPTSF (NY216024), and the support from State Key Laboratory of Environmental Chemistry and Ecotoxicology, Research Center for Eco-Environmental Sciences, Chinese Academy of Sciences (KF2016-05); L. H. Wang acknowledges the National Basic Research Program of China (2012CB933301), the Jiangsu National Synergistic Innovation Center for Advanced Materials 
(SICAM), the Program for Changjiang Scholars and Innovative Research Team in University (IRT_15R37), and the Priority Academic Program Development of Jiangsu Higher Education Institutions (PAPD, YX03001) for support. XCJ acknowledges the Ministry of Education Tier 1 Academic Research Fund (RG131/15) and the support from NTU-Northwestern Institute for Nanomedicine (M4081502.F40).

\section{Notes and references}

1 A. R. Amini, C. T. Laurencin and S. P. Nukavarapu, Crit. Rev. Bioeng., 2012, 40, 363-408.

2 A. J. Salgado, O. P. Coutinho and R. L. Reis, Macromol. Biosci., 2004, 4, 743-765.

3 Y. Liu, J. Lim and S. H. Teoh, Biotechnol. Adv., 2013, 31, 688705.

4 Y. Gao, J. Lim, S.-H. Teoh and C. Xu, Chem. Soc. Rev., 2015, 44, 6306-6329.

5 Y. Liu, J. K. Chan and S. H. Teoh, J. Tissue Eng. Regener. Med., 2015, 9, 85-105.

6 M. B. Nair, J. D. Kretlow, A. G. Mikos and F. K. Kasper, Curr. Opin. Biotechnol., 2011, 22, 721-725.

7 S. Noimark, C. W. Dunnill, M. Wilson and I. P. Parkin, Chem. Soc. Rev., 2009, 38, 3435-3448.

8 R. M. Donlan and J. W. Costerton, Clin. Microbiol. Rev., 2002, 15, 167-193.

9 J. T. Mader, M. Shirtliff and J. H. Calhoun, Best Pract. Res., Clin. Rheumatol., 1999, 13, 1-20.

10 H. W. Kim, J. C. Knowles and H. E. Kim, J. Biomed. Mater. Res., Part B, 2004, 70, 240-249.

11 M. V. Cabanas, J. Pena, J. Roman and M. Vallet-Regi, Eur. J. Pharm. Sci., 2009, 37, 249-256.

12 T. Miyai, A. Ito, G. Tamazawa, T. Matsuno, Y. Sogo, C. Nakamura, A. Yamazaki and T. Satoh, Biomaterials, 2008, 29, 350-358.

13 M. Rai, A. Yadav and A. Gade, Biotechnol. Adv., 2009, 27, 7683.

14 N. Duran, M. Duran, M. B. de Jesus, A. B. Seabra, W. J. Favaro and G. Nakazato, J. Nanomed. Nanotechnol., 2016, 12, 789799.
15 S. Sanyasi, R. K. Majhi, S. Kumar, M. Mishra, A. Ghosh, M. Suar, P. V. Satyam, H. Mohapatra, C. Goswami and L. Goswami, Sci. Rep., 2016, 6, 16.

16 Q. L. Feng, J. Wu, G. Q. Chen, F. Z. Cui, T. N. Kim and J. O. Kim, J. Biomed. Mater. Res., 2000, 52, 662-668.

17 K. B. Holt and A. J. Bard, Biochemistry, 2005, 44, 1321413223.

18 S. Pal, Y. K. Tak and J. M. Song, Appl. Environ. Microbiol., 2007, 73, 1712-1720.

19 J. Ren, W. Wang, S. Sun, L. Zhang, L. Wang and J. Chang, Ind. Eng. Chem. Res., 2011, 50, 10366-10369.

20 A. Tao, P. Sinsermsuksakul and P. D. Yang, Angew. Chem., Int. Ed., 2006, 45, 4597-4601.

21 Y. H. Lee, W. Shi, H. K. Lee, R. Jiang, I. Y. Phang, Y. Cui, L. Isa, Y. Yang, J. Wang, S. Li and X. Y. Ling, Nat. Commun., 2015, 6, 6990.

22 J. Lim, M. S. Chong, J. K. Chan and S. H. Teoh, Small, 2014, 10, 2495-2502.

23 Y. Gao, J. Lim, Y. Han, L. Wang, M. S. Chong, S. H. Teoh and C. Xu, Nanoscale, 2016, 8, 2568-2574.

24 Y. Gao, J. Lim, D. C. Yeo, S. Liao, M. Lans, Y. Wang, S. H. Teoh, B. T. Goh and C. Xu, ACS Appl. Mater. Interfaces, 2016, 8, 6336-6343.

25 Y. Gao, C. Xu and L. Wang, RSC Adv., 2016, 6, 61771-61776. 26 J. H. Shim, M. J. Kim, J. Y. Park, R. G. Pati, Y. P. Yun, S. E. Kim, H. R. Song and D. W. Cho, Tissue Eng. Regener. Med., 2015, 12, 283-293.

27 F. M. Christensen, H. J. Johnston, V. Stone, R. J. Aitken, S. Hankin, S. Peters and K. Aschberger, Nanotoxicology, 2010, 4, 284-295.

28 Z.-Y. Zhang, S. H. Teoh, W.-S. Chong, T.-T. Foo, Y.-C. Chng, M. Choolani and J. Chan, Biomaterials, 2009, 30, 2694-2704.

29 L. Ventrelli, T. Fujie, S. D. Turco, G. Basta, B. Mazzolai and V. Mattoli, J. Biomed. Mater. Res., Part A, 2014, 102, 26522661.

30 C. Wiraja, D. C. Yeo, M. S. K. Chong and C. Xu, Small, 2016, 12, 1342-1350.

31 A. Ravichandran, J. Lim, M. S. K. Chong, F. Wen, Y. Liu, Y. T. Pillay, J. K. Y. Chan and S.-H. Teoh, J. Biomed. Mater. Res., Part B, 2016, DOI: 10.1002/jbm.b.33772. 